\title{
Personality Types, Anxiety and Stress among Young Adults
}

\author{
Sneha K. Sabu ${ }^{1 *}$ and Sannet Thomas ${ }^{2}$
}

${ }^{1} 3^{\text {rd }}$ Year, B.Sc. Psychology Student, Yuvakshetra Institute of Management Studies, Mundoor, Palakkad, Kerala, India

${ }^{2}$ Assistant Professor, Department of Psychology Yuvakshetra Institute of Management Studies, Mundoor, Palakkad, Kerala, India

*Corresponding author: snehaksabu@gmail.com

Received: $19-03-2020$

Revised: 20-06-2020

Accepted: $28-07-2020$

\begin{abstract}
The aim of the study was to analyse the significant difference in the level of Neuroticism, Extraversion, Openness, Agreeableness, Conscientiousness, Anxiety and Stress in accordance to gender and how personality types are related to Anxiety and Stress. Personality is the dynamic organisation within the individual of those psychophysical system that determine his characteristics behaviour and thought (Allport, 1961). Stress is our responses to events that disrupt, our physical and psychological functioning (Lazarus \& Folkman, 1984; Taylor, 1991). Anxiety is the increased arousal accompanied by generalized feelings of fear or apprehension (Baron, 1999). This study was conducted on 120 young adults aged 18 to 26 years, out of which 60 were males and 60 were females, through purposive sampling technique. The Neo Five Factor Inventory (Costa, 1978), Beck Anxiety Inventory (Beck, 1993) and the Perceived Stress Scale (Cohen, 1983) were used to collect data. Data were analysed using mean, one-way ANOVA and Pearson's product moment correlation. Result proves that, there is no significant difference in Neuroticism, Extraversion, Openness, Agreeableness, Conscientiousness, Anxiety and Stress among young adults across gender. There is a correlation between Neuroticism and Stress among young adults. There is a correlation between Neuroticism and Anxiety among young adults. There is a correlation between Conscientiousness and Anxiety among young adults. There is a correlation between Anxiety and Stress among young adults.
\end{abstract}

Keywords: Personality, Neuroticism, Extraversion, Openness, Agreeableness, Anxiety, Stress

Personality can be defined as the distinctive and characteristic patterns of thought, emotion, and behavior that make up an individual's personal style of interacting with the physical and social environment (Atkinson \& Hilgard, 2009). Personality constitutes of an individual's traits or characteristics that are relatively stable and unique. Many psychologists have explained behavioural differences among individuals and consistencies within an individual, through many approaches and theories. Characterising personality helps predict how they will behave in certain situations and also helps to deal with them more effectively. Even though the differences are of wide range and each individual is unique, we can still club them into a smaller number of personality traits, on the basis of primary traits. One such classification is the Five Factor Model by Robert McCrae and Paul Costa. They identified five robust factors, they are: Neuroticism, Extraversion, Openness, Agreeableness and Conscientiousness. Neuroticism compares adjustment or emotional stability with maladjustment or neuroticism. Neurotic personalities have a general tendency to experience negative effects such as fear, sadness, embarrassment, anger, guilt and disgust. They are also disposed to to have irrational ideas, lower impulse control, and to cope poorly with stress. Extraversion comprises of sociable, assertive, active

How to cite this article: Sabu, S.K. and Thomas, S. (2020). Personality Types, Anxiety and Stress among Young Adults. Educational Quest: An Int. J. Edu. Appl. Soc. Sci., 11(2): 125-131.

Source of Support: None; Conflict of Interest: None ⿷匚⿳丨コ丨 
and talkative, they are fun loving, upbeat and optimistic. Introverts are reserved, independent and even paced. Openness consist of creativity, curiosity, aesthetic and preference of variety. Individuals high on openness tend to react to originality. Agreeableness encompasses traits such as courtesy, politeness, altruistic, sympathetic and eager to help. Disagreeable people tend to be egocentric. Conscientiousness refers to people who are reliable, organized, punctual, scrupulous, they tend to be workaholic and unbearably neat. Anxiety involves a general feeling of apprehension about possible future danger, it is a complex blend of unpleasant emotions and cognitions that is both oriented to the future (Butcher, 2016). Anxiety negative mood, self-preoccupation, feeling unable to control the future, a sense of tension, over arousal. Anxiety in an adaptive way helps us to prepare for a possible threat but extreme level of anxiety to a situation may reveal a problem. People with anxiety-related problems feel threatened, but they don't do anything constructive about it. They struggle to control themselves, but they remain ineffective and unhappy (Rachman, 2004). Anxiety becomes problematic when it prevents people from doing what they want or need to do and when people simply cannot stop worrying. Stress can be described as the difficulties and strains experienced by living organisms as they struggle to cope with and adapt to changing environmental conditions (Selye.1976). Stress increases the probability of one's survival, it motivates and causes arousal which affects the performance of an individual. An optimal level of stress i.e., the eustress is very important. In contrast, increased level of stress can cause damage. An individual's response to a stressful situation is largely influenced by how they interpret or judge a perceived situation.

\section{Relevance of the study}

This Study on Personality Types, Anxiety and Stress among Young Adults is relevant because it tries to comprehend the relationship between personality types, stress and anxiety among young adults. This study was conducted to understand whether there are any differences in how different types of personality cope with stress and anxiety. This study was conducted during the COVID - 19 outbreaks, thus, the study has its relevance in understanding how young adults in Kerala were coping and adjusting with this unpredictable and uncontrollable situation i.e., the lockdown. Different personality types show different level of copying with stress and anxiety, this study was helpful in understanding those differences.

\section{Definition of key terms}

Personality is the dynamic organisation within the individual of those psychophysical system that determine his characteristics behaviour and thought (Allport, 1961).

Stress is our responses to events that disrupt, our physical and psychological functioning (Lazarus \& Folkman, 1984; Taylor, 1991).

Anxiety is the increased arousal accompanied by generalized feelings of fear or apprehension (Baron, 1999).

\section{Review of Literature}

Ann-Christine Andersson Arntenl, Bengt Janssonl and Trevor Archerl conducted a study on Influence of Affective Personality type and Gender upon Coping Behavior, Mood and Stress in 2008. Two studies were carried out, in Study 1 involving 75 participants and In Study 11, involving 139 participants from different occupations, type of affective personality was influenced by individuals' expressions of P A, NA, cognitive, emotional, physical, social and spiritual coping, dispositional optimism, anxiety and depression, stress, work stress and partnership relations. Both study indicated that affective personality type was associated with individuals' levels of depression and anxiety, as well as stress and energy which contributes to an individual's ability to deal with life situations.

In 2010, Pedro González Leandro and M Dolores Castillo conducted a study on Coping with stress and its relationship with personality dimensions, anxiety, and depression. The sample was comprised of 274 participants ( $44 \%$ male and $56 \%$ female) with an age range of 17 to 62 years old. Differences in coping styles between groups of participants with both high and low levels in different dimensions of personality were found. Furthermore, it was observed that gender modulates the coping styles used (for example, although task-focused coping is 
used equally by both genders, the emotion-focused coping is used more by women). Considering overall measures of coping styles, the results indicate that task-focused coping is used more by participants with low external locus of control, high self-esteem, and low anxiety and depression. On the contrary, the emotion-focused coping is used more by participants with high external locus of control, low self-esteem, and high depression. However, gender exerts a modulating effect on these result.

Thomas Olsen Gramstad, Rolf Gjestad and Brit Haver conducted a study on Personality traits predict job stress, depression and anxiety among junior physicians in 2013. Two full classes of medical students $(n=281)$ at the University of Bergen, Norway participated in a mandatory intervention program during their third undergraduate year. The study indicated that There is a relation between certain personality traits measured early in medical school and later mental health among junior physicians in internship training following graduation from the University of Bergen.

\section{METHODS}

\section{Problem}

Is there any significant difference between personality types, the level of anxiety and stress among young adults across gender?

\section{Aim}

To understand how personality types are related to anxiety and stress differ in young adults across gender.

\section{Objectives}

1. To study the personality types, anxiety and stress among young adults based on gender.

2. To compare anxiety, stress and personality types young adults.

\section{Variables}

Independent variable: Gender

Dependent variable: Personality types, Anxiety, Stress

\section{Hypothesis}

In accordance to the above mentioned objectives, the following 14 hypotheses were formulated:
1. There will be no significant difference in Anxiety among young adults across gender.

2. There will be no significant difference in Stress among young adults across gender.

3. There will be no significant difference in Neuroticism and Stress among young adults.

4. There will be no significant difference in Extraversion and Stress among young adults.

5. There will be no significant difference in Openness and Stress among young adults.

6. There will be no significant difference in Agreeableness and Stress among young adults.

7. There will be no significant difference in Conscientiousness and Stress among young adults.

8. There will be no correlation between Neuroticism and Stress among young adults.

9. There will be no correlation between Extraversion and Stress among young adults.

10. There will be no correlation between Openness and Stress among young adults.

11. There will be no correlation between Agreeableness and Stress among young adults.

12. There will be no correlation between Conscientiousness and Stress among young adults.

13. There will be no correlation between Neuroticism and Anxiety among young adults.

14. There will be no correlation between Extraversion and Anxiety among young adults.

15. There will be no correlation between Openness and Anxiety among young adults.

16. There will be no correlation between Agreeableness, and Anxiety among young adults.

17. There will be no correlation between Conscientiousness and Anxiety among young adults.

18. There will be no correlation between Anxiety and Stress among young adults. 


\section{Research Design}

The study was a quantitative study and used correlation to determine the relationship between personality types, anxiety and stress among young adults. The study also adopted between-group design to assess the difference in personality types, anxiety and stress among young adults based on gender.

\section{Research Sample}

Purposive sampling method was used to collect data in this study. The study consisted of 120 samples out of which 60 were males and 60 were females. The sample belonged to different religious, family types, economy and area of residence. The data was collected from young adults aged 18 to 26 years.

\section{Sampling criteria}

\section{Inclusion criteria}

1. Willingness of the participants via online

\section{Exclusion criteria}

2. Age group other than $18-26$ years

\section{Tools}

The following tools were administered individually to collect the required data:

1. The Neo Five Factor Inventory (Costa, 1978).

2. Beck Anxiety Inventory (Beck, 1993).

3. Perceived Stress Scale (Cohen, 1983).

\section{Procedure}

The purpose of the study was explained to the participants and their willingness to participate in the study was ascertained through online. The sociodemographic sheet, The Neo Five Factor Inventory (Costa, 1978), Beck Anxiety Inventory (Beck, 1993) and the Perceived Stress Scale (Cohen, 1983) were provided to the participants through online forms. Proper instructions were given before each questionnaire. Responses were collected from the participants. The scoring and interpretation of the responses were done according to the manual.

\section{Analysis of Data}

The collected data was analysed using the Statistical
Package for Social Science (SPSS) version 20. The following statistical tests were used:

1. Mean, Standard Deviation

2. One-way ANOVA

3. Pearson's product moment correlation.

\section{Ethical issues}

1. Confidentiality, of the information obtained from the participants, was maintained throughout the study.

2. The collected data has been used only for research purpose.

3. Informed consent was taken from the participants, through online, before collecting the data.

\section{RESULTS}

\section{Data Analysis and its Interpretation}

The main purpose of the study was to investigate the personality types, anxiety and stress among young adults in Kerala. For this purpose, 6 hypotheses were formulated. Results are shown in the tables given below.

Table 1 shows the gender difference on personality types, anxiety and stress, and the data was analysed through one-way ANOVA. The analysis of data show females have high level of neuroticism, extraversion, openness agreeableness and conscientiousness compare to males and, the males have high level of anxiety and stress compared to females, but it is not statistically proven. So, the null hypothesis 1,2 and 3 were accepted. That is, there is no significant difference in the Personality Types among young adults across gender, there is no significant difference in Anxiety among young adults across gender and there is no significant difference in Stress among young adults across gender.

According to Table 2, the null hypotheses 4, 9,13 and 14 were rejected and the result show that there is a correlation between Neuroticism and Stress among youth, there is a correlation between Neuroticism and Anxiety among youth, there is a correlation between Conscientiousness and Anxiety among youth and there is a correlation between Anxiety and Stress among youth. The null hypotheses 5 6, 7, $8,, 10,11$ and 12 were accepted and the result show 
Table 1: One way ANOVA neuroticism, extraversion, openness, agreeableness, conscientiousness, anxiety and stress by gender(between group)

\begin{tabular}{|c|c|c|c|c|c|c|c|}
\hline Variable & Gender & $\mathbf{N}$ & Mean & SD & SS & Df & Sig. \\
\hline \multirow[t]{3}{*}{ Neuroticism } & Male & 60 & 24.3333 & 5.14754 & & & \\
\hline & Female & 60 & 25.4000 & 5.83153 & 34.133 & 1 & .290 \\
\hline & & 120 & & & & & \\
\hline \multirow[t]{3}{*}{ Extraversion } & Male & 60 & 27.4667 & 5.29940 & & & \\
\hline & Female & 60 & 28.2833 & 4.30251 & 0.008 & 1 & .356 \\
\hline & & 120 & & & & & \\
\hline \multirow[t]{3}{*}{ Openness } & Male & 60 & 25.8500 & 4.47147 & & & \\
\hline & Female & 60 & 27.3000 & 4.98234 & 63.075 & 1 & .096 \\
\hline & & 120 & & & & & \\
\hline \multirow[t]{3}{*}{ Agreeableness } & Male & 60 & 25.6833 & 4.82048 & & & \\
\hline & Female & 60 & 25.8333 & 4.76984 & .675 & 1 & .864 \\
\hline & & 120 & & & & & \\
\hline \multirow[t]{3}{*}{ Conscientiousness } & Male & 60 & 27.5333 & 4.79218 & & & \\
\hline & Female & 60 & 28.5833 & 5.03981 & 33.075 & 1 & .245 \\
\hline & & 120 & & & & & \\
\hline \multirow[t]{3}{*}{ Anxiety } & Male & 60 & 16.6333 & 12.24463 & & & \\
\hline & Female & 60 & 15.4333 & 9.83140 & 43.200 & 1 & .555 \\
\hline & & 120 & & & & & \\
\hline \multirow[t]{3}{*}{ Stress } & Male & 60 & 20.2000 & 6.19677 & & & \\
\hline & Female & 60 & 19.5000 & & 14.700 & 1 & .478 \\
\hline & & 120 & & & & & \\
\hline
\end{tabular}

Table 2: correlations between variables

\begin{tabular}{llll}
\hline & & Anxiety & Stress \\
\hline Neuroticism & Pearson Correlation & .398 & .571 \\
& Sig. (2-tailed) & .000 & .000 \\
Extraversion & $\mathrm{N}$ & 120 & 120 \\
& Pearson Correlation & -.110 & -.165 \\
& Sig. (2-tailed) & .234 & .071 \\
Openness & $\mathrm{N}$ & 120 & 120 \\
& Pearson Correlation & -.042 & -.166 \\
Agreeableness & Sig. (2-tailed) & .651 & .069 \\
& $\mathrm{~N}$ & 120 & 120 \\
Conscientiousness & Pearson Correlation & -.157 & -.174 \\
& Sig. (2-tailed) & .087 & .057 \\
Anxiety & $\mathrm{N}$ & 120 & 120 \\
& Pearson Correlation & -.089 & -.199 \\
Stress & Sig.(2-tailed) & .333 & .030 \\
& $\mathrm{~N}$ & 120 & 120 \\
& Pearson Correlation & 1 & .493 \\
& Sig.(2-tailed) & 120 & .000 \\
& $\mathrm{~N}$ & & 120 \\
\hline & Pearson Correlation & 493 & 1 \\
& Sig.(2-tailed) & .000 & 120 \\
$\mathrm{~N}$ & 120 & \\
\hline
\end{tabular}


that there is no correlation between Extraversion and Stress among young adults, there is no correlation between Openness and Stress among young adults, there is no correlation between Agreeableness and Stress among young adults, there is no correlation between Conscientiousness and Stress among young adults, there is no correlation between Extraversion and Anxiety among young adults, there is no correlation between Openness and Anxiety among young adults, and there is no correlation between Agreeableness, and Anxiety among young adults.

\section{Major Findings}

1. There is no significant difference in Anxiety among young adults across gender.

2. There is no significant difference in Stress among young adults across gender.

3. There is no significant difference in Neuroticism and Stress among young adults.

4. There is no significant difference in Extraversion and Stress among young adults.

5. There is no significant difference in Openness and Stress among young adults.

6. There is no significant difference in Agreeableness and Stress among young adults.

7. There is no significant difference in Conscientiousness and Stress among young adults.

8. Females have high level of Neuroticism compared to males.

9. Females have high level of Extraversion compared to males.

10. Females have high level of Openness compared to males.

11. Males and females have comparatively equal level of Agreeableness.

12. Females have high level of Conscientiousness compared to males.

13. Males have high level of Anxiety compared to females.

14. Males have high level of Stress compared to females.

15. There is a correlation between Neuroticism and Stress among young adults.

16. There is no correlation between Extraversion and Stress among young adults.
17. There is no correlation between Openness and Stress among young adults.

18. There is no correlation between Agreeableness and Stress among young adults.

19. There is no correlation between Conscientiousness and Stress among young adults.

20. There is a correlation between Neuroticism and Anxiety among young adults.

21. There is no correlation between Extraversion and Anxiety among young adults.

22. There is no correlation between Openness and Anxiety among young adults.

23. There is no correlation between Agreeableness, and Anxiety among young adults.

24. There is a correlation between Conscientiousness and Anxiety among young adults.

25. There is a correlation between Anxiety and Stress among young adults.

\section{Limitations}

The sample size was small.

Only quantitative research method was used.

The sample size was restricted to a specific age group only.

The researcher has not taken other domains such as socio-economic status, family type, etc. which also may influence personality types, anxiety and stress.

$\square$ As the study was conducted via online survey forms, the genuineness and seriousness of the participants, while answering cannot be ensured.

\section{Implications}

As the study indicated that males have high level of anxiety and stress compared to females, we can use some intervention programmes.

$\square$ The study indicated that females have high level of neuroticism compared to males, intervention programmes can be done among females.

$\square$ As the study indicated that males have low level of openness and conscientiousness compared to females, some intervention programmes can be used. 
And as the study shows a positive correlation between some personality types (neuroticism and conscientiousness) and, anxiety and stress. The study can be useful for the welfare of people high on Neuroticism and Conscientiousness, as they are more prone to anxiety and stress compared to the other personality types.

\section{Scope for further study}

Further study can be done on a large sample.

$\square$ Qualitative method can be used to improve the quality of the study.

\section{REFERENCES}

Arnten, A.A., Jansson, B. \& Archer, T. 2008. Influence of Affective Personality type and Gender upon Coping Behavior, Mood and Stress. Individual Differences Research, 6(3): 139-168.
Baron, R.A. \& Misra, G. 2016. Psychology, Indian subcontinent edition. ( $5^{\text {th }}$ ed.), Pearson Education.

Butcher, J.N., Hooley, J.M. \& Mineka, S. 2019. Abnormal Psychology (17 $7^{\text {th }}$ ed.), Pearson Education.

Coon, D. \& Mitterer, J.O. 2007. Introduction to Psychology, gateways to mind and behavior ( $11^{\text {th }}$ ed.), Thomson Wadsworth.

Gramstad, T.O., Gjestad, R. \& Haver, B. 2013. Personality traits predict job stress, depression and anxiety among junior physicians. BMC Medical Education, 13(1).

Leandro, P.G. \& Castillo, M.D. 2010. Coping with stress and its relationship with personality dimensions, anxiety, and depression. Procedia Social and Behavioral Sciences, 5(1).

Schultz, D.P. \& Schultz, S.E. 2004. Theories of personality $\left(8^{\text {th }}\right.$ ed.) Thomson Wadsworth. 
